\title{
Specifying Information Security Needs for the Delivery of High Quality Security Services
}

\author{
Xiaomeng Su \\ Telenor R\&I \\ 7004 Trondheim, Norway \\ Email: xiaomeng.su@telenor.com
}

\author{
Damiano Bolzoni \\ University of Twente \\ 7500 AE Enschede, The Netherlands \\ Email: damiano.bolzoni@utwente.nl
}

\author{
Pascal van Eck \\ University of Twente \\ 7500 AE Enschede, The Netherlands \\ Email: p.a.t.vaneck@utwente.nl
}

\begin{abstract}
In this paper we present an approach for specifying and prioritizing information security requirements in organizations. We propose to explicitly link security requirements with the organization's business vision, i.e. to provide business rationale for security requirements. The rationale is then used as a basis for comparing the importance of different security requirements. Furthermore we discuss how to integrate the aforementioned solution concepts into a service level management process for security services, which is an important step in IT Governance.
\end{abstract}

\section{INTRODUCTION}

It is clear that senior managers in many organizations are now expressing a much greater interest in information security [1]. Understanding and specifying what kind of security an organization need is however a difficult task. Many underlying goals (why and what security is needed) remain tacit within organizations and requirements end up being articulated as specifications of the security control baseline (how security will be achieved) without a clear rationale. The aim of this paper is to develop techniques and instruments to help stakeholders articulate the connection between security requirements and business drivers in a systematic way. This connection is needed to provide the rationale to prioritize security requirements. The reason of making explicit the business rationale behind security requirement is twofold. Firstly, it forces the stakeholders to turn their intuition into explicit judgments - judgments that are based on business goals and whose underlying assumptions are discussed openly. Secondly, since hundred per cent security is not achievable and the limited resources available should be directed to satisfy the most important ones, we need a way to prioritize security requirements. The business rationale serves as the underlying criterion for evaluating how important each security requirement is.

\section{Formulating AND Understanding SECURITy GOALS AND REQUIREMENTS}

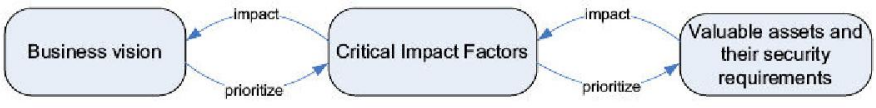

Fig. 1. Linking security requirements with business vision via CIF.

A security requirement specification tells what should be secured and why. It identifies the organization's needs with respect to security. When an organization wants to secure its systems, it must first determine what requirements to meet. Given that organizations normally have limited resources to protect their assets, it is equally important to determine which requirements are more important and thus should be prioritized. To achieve this, we propose to use a conceptual framework where security requirements are linked to the unique business drivers of the organization in question. Figure 1 portrays the conceptual framework. The business vision consists of high level business goals the organization has. Critical Impact Factors (CIFs) identify what will be the business impacts if security requirements are violated. Valuable assets and their security requirements are inventories of security requirements. Valuable assets and their security requirements have an effect on the CIFs and the CIFs in turn impact the accomplishment of the organization's business vision. In other words, we can use an organization's business vision to prioritize the CIFs, which can be used to further prioritize the security requirements. To achieve that, three subsequent steps need to be taken. Firstly an organization's CIFs and business vision need to be defined. Secondly, we need to enumerate valuable assets and their security requirements. Thirdly, security requirements shall be linked with CIFs and business vision. For a detailed illustration of the framework, we refer readers to [2].

\section{INTEGRATING THE SOLUTION CONCEPTS INTO SERVICE LEVEL MANAGEMENT CyCle}

The solution concepts presented in the previous section are of practical usage only when they can be integrated into processes and activities conducted in an organization. In this section, we discuss how our solution concepts can be integrated in service level agreements (SLAs) in the context of IT governance.

Figure 2 shows an abstract process model taken from [3], which describes the process of managing and maintaining service level agreements. In Figure 2, it has been adapted to the security domain. The left part of the lemniscate concerns the specification of security services (upper arrow) and the evaluation and monitoring of the performance of the service provider (lower arrow). The right part concerns the evaluation and monitoring of security service processes (upper arrow) and the design and organization of those processes. The service 


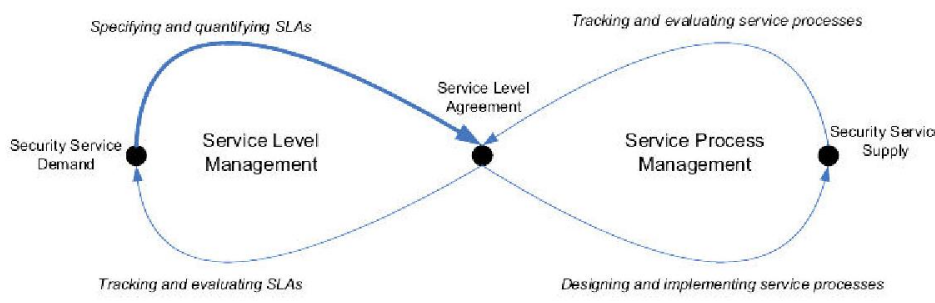

Fig. 2. Security service level management lemniscate adapted from [3].

level management lemniscate is an abstract model that needs to be refined for the particular case at hand. Figure 3 shows our proposed refinement, in which the upper-left arrow of Figure 2 has been refined into seven process steps. The vertical dimension depicts the distinction between the IT demand side of an organization (Business, i.e. those organizational units where IT is used but not provided) and the IT supply side (IT, i.e. those organizational units that provide IT services). The figure shows that in our vision, the need for security services originate at the demand side, in the sense that it is ultimately the demand side that is harmed by breaches resulting from lack of security. It is also the demand side that is responsible for determining the business vision and critical impact factors (but often, the IT supply side provides help in carrying out these steps). The IT side then takes over, and the whole process results in SLAs that formalize the relation between the demand side and supply side.

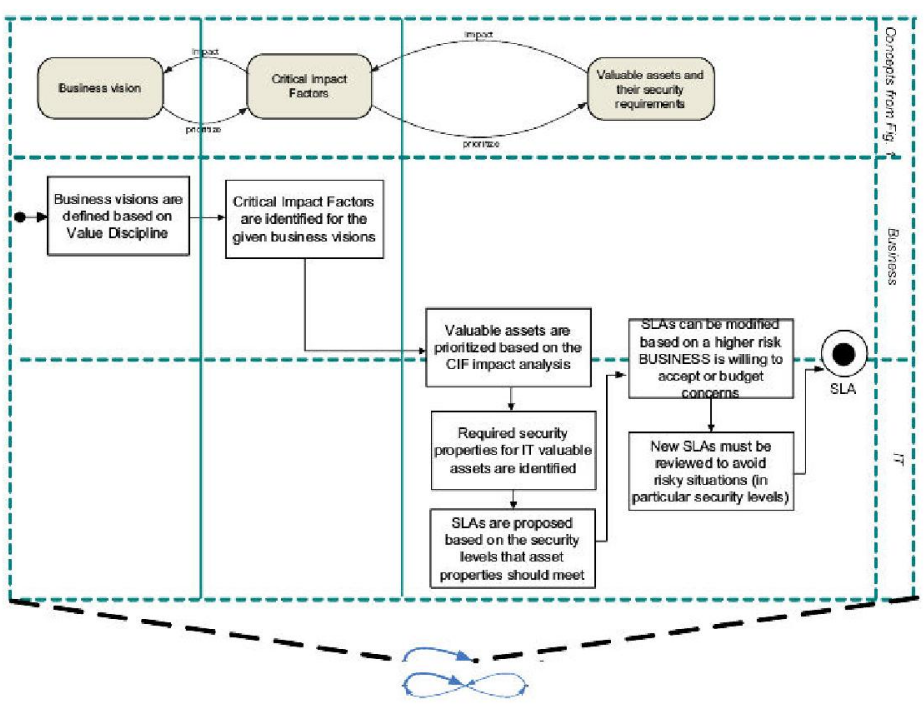

Fig. 3. Steps for structured translation of IT security needs into SLAs

The steps for specifying and quantifying SLAs for security services illustrated in Figure 3 are as follows: 1) The business unit starts to define its business vision by the help of identifying its value discipline. If the business vision already exist as a result of earlier activities, this step can be omitted. 2) Critical Impact Factors are identified based on a combination of industry specific CIFs, reviews of peer CIFs and the organization's own security officer's input. 3) The valuable business assets are listed and prioritized using the CIF impact analysis. This step is performed by the business unit and IT security unit together. 4) For the prioritized business assets, IT security unit identifies the IT assets that are needed to support the business assets and further identifies the desired security properties for the IT assets. 5) SLAs are prepared by IT and proposed to the business unit. 6) Due to budget concerns and maybe other causes, business may decide to accept a higher risk for certain assets in return for a lower service price. 7) Finally, the new SLAs must be reviewed by IT to make sure that the higher risks in one business unit does not impose unacceptable high risks to other business units. This could happen because of the inter-dependence of processes across business units.

\section{CONCLUSIONS}

Information security is ultimately about business security. In this paper, we have proposed a conceptual framework that makes the link between security requirements and the organization's business drivers explicit. The three main elements of our framework are business vision, CIFs and valuable assets and their security requirements. The connection between business goals and security requirements, once established, can be used to provide rationale for prioritizing security requirements. The conceptual framework presented in this paper is only useful in practice when it is embedded in a concrete process for security management. In this paper, we have shown how this can be accomplished in the context of IT service level management. We have evaluated our approach by means of a focus group session at a large financial institution. Our experience with the focus group session tells us that giving people the appropriate tools to frame and structure their decision making process in relation to the underlying business goals and encouraging the right kind of dialog among stakeholders are beneficial to the delivery of high quality services. From what we have learned, several directions for further research become apparent. For example, our focus group indicated that creating security awareness is a very critical success factor. It is also our intention to extend our security management process to cover tracking and evaluating service level agreements.

a) Acknowledgments: We thank our focus group at Bank A for their valuable contribution.

\section{REFERENCES}

[1] L. A. Gordon, M. P. Loeb, W. Lucyshyn, and R. Rochardson, "2005 CSI/FBI computer crime and security survey," Computer Security Institute, Tech. Rep., 2005

[2] X. Su, D. Bolzoni, and P. A. T. van Eck, "A business goal driven approach for understanding and specifying information security requirements," in 11th International Workshop on Exploring Modeling Methods in Systems Analysis and Design (EMMSAD2006), Luxembourg. Presses Universitaries de Namur, 2006, pp. 465-472.

[3] F. Niessink, "Perspectives on improving software maintenance," Ph.D. dissertation, Division of Mathematics and Computer Sciences, Faculty of Sciences, Vrije Universiteit, Amsterdam, the Netherlands, March 2000. 